\title{
Fabrication of bicomponent nanoantennas interfaces by femtosecond laser ablation method
}

\author{
Ruslan Chkalov*, Dmitriy Kochuev, Kirill Khorkov, and Valery Prokoshev \\ Vladimir State University, 600000 Vladimir, Russia
}

\begin{abstract}
The work is devoted to the problem of fabrication interfaces of bicomponent nanoantennas. The possibility of using controlled laser ablation technology for the formation of thin-film elements is described. The applicability of the approach for rapid prototyping of various geometric configurations of optical antennas interfaces is shown.
\end{abstract}

\section{Introduction}

Optical antennas are nanoscale elements, which characteristic feature is the ability to amplify photophysical phenomena. This property makes nanoantennas an extremely promising elements for use in optoplasmonics for solving problems of generating surface plasmon polaritons. Such antennas are two-component structures that include a dielectric substrate covered with a thin metal film [1]. Physical properties of the antenna depends on the type of metal used, the spatial geometry, the width of the gap between the nanostructures, the radius of surface curvature. Thus, changing the parameters of antenna interfaces allows to control the position of frequencies at which the maximum amplification of local field strength is observed, which determines the working emission spectrum and the target applications of created elements.

\section{Laser writing of thin-film interfaces}

An actual approach to solving the problem of producing nanocircuit elements, in particular, nanoantennas interfaces, is the use of femtosecond laser writing technology to form the topological structure of elements on a substrate surface. Characteristic features of laser pulses with a duration of less than picoseconds are the ultrafast transfer of radiation energy to a material being processed and extremely low heat removal from the impact zone, which allows to minimize an occurrence of thermal distortion [2]. These conditions allow the use of a much lower average radiation power and pulse energy for removal of material areas, due to localization in a strictly limited area of the laser spot. The technology of selective laser ablation of thin-film coatings makes it possible to achieve high accuracy in the formation of

\footnotetext{
* Corresponding author: j.larenax@gmail.com
} 
nanoantennas topologies, at the same time, some geometric parameters of elements can reach nanometer order.

Selective laser removal of film areas is carried out by moving the sample relative to a stationary laser beam with a given speed (Fig. 1), which implies the need for high resolution spatial positioning of the sample and accurate synchronization with the laser system. The presented technique allows to form basic types of plasmon nanoantenna interfaces (with a flat tip, a tapered tip, a bow-tie) [3,4], and combined experimental interfaces of complex geometry. Ultrashort laser pulses make it possible to process almost any material, including coatings that are most often used to form a conductive layer of a metal substrate: gold, silver, copper.

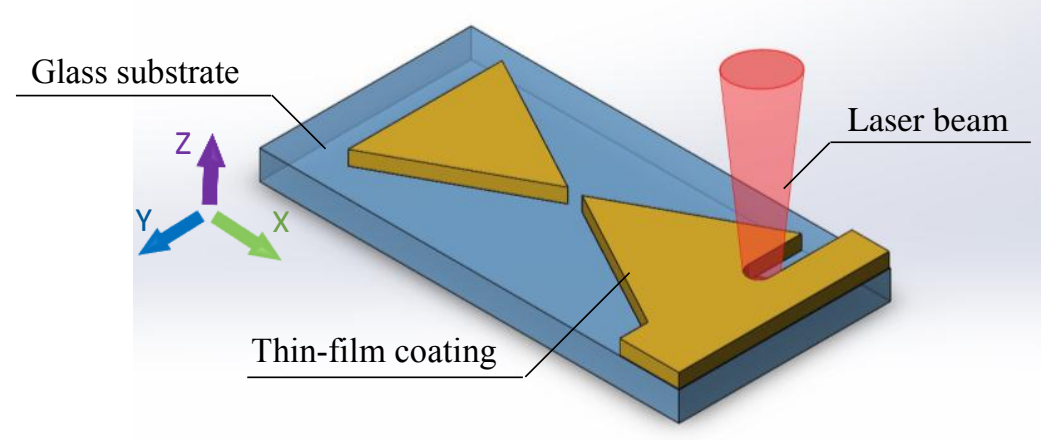

Fig. 1. The principle of nanoantennas interfaces formation by controlled laser ablation technology.

The use of ultrashort laser pulses for selective removal of thin film coatings offers a unique approach for rapid prototyping and testing of various geometric configurations of optical antennas interfaces. Properly selected exposure mode allows to avoid physical and thermal damage of surrounding areas, and occurrence of negative changes in dielectric substrate morphology.

\section{Conclusion}

At the moment, nanoantennas have already found their application in many high-tech fields and are successfully used to solve tasks of near-field microscopy, Raman spectroscopy, and high-resolution biomedical sensors development [5]. Taking into account the diversity of sources and detectors of highly localized fields, it can be argued that the area of practical applicability of nanoantennas in the near future is comparable with their classical counterparts.

\section{References}

1. A. E. Krasnok, I. S. Maksymov, A. I. Denisyuk, P. A. Belov, A. E. Miroshnichenko, C. R. Simovski, Y. S. Kivshar, Phys. Usp., 56(6), 539 (2013).

2. R. Chkalov, K. Khorkov, D. Kochuev, Mater. Tod. Proc., 11, 441 (2019).

3. F. Laible, D. A. Gollmer, S. Dickreuter, D. P. Kern, M. Fleischer, Nanosc., 10(31), 14915-14922 (2018).

4. M. J. Madou, CRC press, 2 (2011).

5. F. Yesilkoy, R A. Terborg, J. Pello, A. A. Belushkin, Y. Jahani, V. Pruneri, H. Altug, Light. Science. Applic., 7 (2018). 